\title{
Elimination of neglected tropical diseases under preventive chemotherapy programmes in the Eastern Mediterranean Region ${ }^{1}$
}

Citation: Elimination of neglected tropical diseases under preventive chemotherapy programmes in the Eastern Mediterranean Region. East Mediterr Health J. 2020;26(12):1576-1577 https://doi.org/10.26719/2020.26.12.1576

Copyright @ World Health Organization (WHO) 2020. Open Access. Some rights reserved. This work is available under the CC BY-NC-SA 3.0 IGO license (https://creativecommons.org/licenses/by-nc-sa/3.o/igo).

\section{Introduction}

The World Health Organization (WHO) global and regional preventive chemotherapy programmes have made a number of key accomplishments. These include the elimination of lymphatic filariasis as a public health problem in Yemen and the establishment of public-private partnerships to facilitate progress towards the elimination and control of neglected tropical diseases (NTDs) (1). More than 1 billion people had been treated globally in 2018 for at least one of five NTDs targeted for control and elimination, with over 1.7 billion treatments distributed to populations in need through mass drug administration (MDA) and a regional coverage of preventive chemotherapy of $21.4 \%$ (2).

As an important step in paving the way to end the epidemics of NTDs by 2030, the 18th meeting of the Regional Programme Review Group (RPRG) on elimination of neglected tropical diseases under preventive chemotherapy programmes in the WHO Eastern Mediterranean Region was convened by the WHO Regional Office for the Eastern Mediterranean in Sharm El Sheikh, Egypt, 9-11 December 2019 (3). The meeting was attended by the members of the RPRG, representatives from the ministries of health of Afghanistan, Djibouti, Egypt, Iraq, Morocco, Pakistan, Saudi Arabia, Somalia, Sudan, Syrian Arab Republic, Tunisia and Yemen. Representatives of partner organizations, including the Expanded Special Project for Elimination of Neglected Tropical Diseases (ESPEN), Mectizan Donation Program, Sightsavers and United Nations Relief and Works Agency for Palestine Refugees in the Near East (UNRWA) also attended the meeting.

The objectives of the meeting were to:

- review country-specific progress made during 2018 and 2019 by preventive chemotherapy programmes for NTDs to track the progress made towards achieving the NTD milestones and targets included in the roadmap for WHO's work in the Eastern Mediterranean Region, discuss challenges in implementation and identify solutions;

- update participants on the current situation, innovations and challenges for control and elimination of NTDs, with a focus on lymphatic filariasis, schistosomiasis, onchocerciasis, soil-transmitted helminthiasis and trachoma in the Region;

- present the WHO NTD roadmap 2030 and discuss its adaptation to country context and the development of country-specific NTD plans for 2021-2030; and

- discuss country-specific plans of action for 2020, including drug requirements, to provide input and recommendations on their funding, design and implementation.

\section{Summary of discussions}

A global update on NTDs was presented along with progress towards achieving NTD targets in the Eastern Mediterranean Region (EMR). In 2017, the size of the population in the EMR requiring interventions against NTDs was estimated at 10 million less than in 2016, mainly due to the reduction of soil-transmitted helminthiasis prevalence in some areas of Sudan as indicated by a prevalence survey conducted in 2017. In addition, The Egyptian Ministry of Health and Population has accelerated elimination of schistosomiasis (commonly known as bilharziasis) by earmarking an equivalent of US\$ 2 million a year for a period of five years (4).

The NTD Roadmap 2021-2030 was presented, which is a high-level global strategy that will set the overall direction for the fight against NTDs, tailored to a diverse set of audiences. The content of the roadmap was outlined, including the milestones for overarching and cross-cutting targets, the disease-specific targets and an analysis of those areas requiring strengthening across multiple diseases.

The process of validation of trachoma elimination as a public health problem was discussed. In addition, the WHO Joint Application Package for preventive chemotherapy was presented and common mistakes, challenges and shortfalls when preparing the Joint Reporting Forms and Joint Request for Selected Medicines were explained.

\section{Recommendations}

\section{To WHO}

- Providing technical support and capacity-building to countries for developing/updating NTD elimination and control plans and for their implementation;

\footnotetext{
This summary is extracted from the Summary report on the Eighteenth meeting of the Regional Programme Review Group on the elimination of neglected tropical diseases under preventive chemotherapy programmes in the Eastern Mediterranean Region, Sharm el Sheikh, Egypt, 9-11 December 2019 (https://applications.emro.who.int/docs/WHOEMCTD083E-eng.pdf?ua=1).
} 
- assisting countries to identify local research priorities for overcoming operational challenges;

- advocating for NTD programmes and resource mobilization initiatives with senior health and finance ministry officials to ensure the allocation of resources from domestic funding and partners;

- maintaining annual RPRG/programme managers' meetings to provide an opportunity for Member States to obtain new information, learn best practices and share experiences with all stakeholders, including donors; and

- facilitating cross-border collaboration and coordination between neighbouring countries and regions, where appropriate.

\section{To Member States}

- Strengthening national NTD control/elimination programmes and having focal persons at national and subnational levels to coordinate activities;

- establishing a multisectoral coordination mechanism/ committee for NTDs, including representation from relevant sectors and stakeholders, such as the finance, education and water, sanitation and hygiene sectors;

- integrating NTD interventions within existing health programmes that receive substantial funding support; and

- developing a compelling evidence-based investment case that NTD elimination and control supporting inclusion within national health plans and policy.

\section{References}

1. World Health Organization. Despite challenges, Yemen eliminates lymphatic filariasis. Geneva: World Health Organization; 2019 (https://www.who.int/neglected_diseases/news/yemen-eliminates-lymphatic-filariasis/en/).

2. World Health Organization. Neglected tropical diseases: treating over one billion people for the fourth successive year. Geneva: World Health Organization; 2019 (https://www.who.int/neglected_diseases/news/treating-over-one-billion-people-for-the-fourthsuccessive-year/en/).

3. World Health Organization Regional Office for the Eastern Mediterranean (WHO/EMRO). Eighteenth meeting of the Regional Programme Review Group on the elimination of neglected tropical diseases under preventive chemotherapy programmes in the Eastern Mediterranean Region, Sharm el Sheikh, Egypt, 9-11 December 2019 (https://applications.emro.who.int/docs/ WHOEMCTDo83E-eng.pdf?ua=1).

4. World Health Organization. Egypt leverages domestic funding to eliminate schistosomiasis. Geneva: World Health Organization; 2016 (https://www.who.int/neglected_diseases/news/Egypt_leverages_domestic_funding_to_eliminate_schistosomiasis/ en/). 